\title{
Intelligent Information Processing in the Problem of Propulsion
}

論文

Paper

\section{System Condition Monitoring*}

\author{
Oleksiy Bondarenko** Tetsugo Fukuda** Ken Miyachi***
}

\begin{abstract}
During the past years, in response to the gradually stringent regulations, the undertaken steps in the shipping industry spurred the technological improvement in ship and engine designs. Thus, new engines accept a wide variety of fuels, and electronic systems provide flexible control and online tuning. At the same time, simultaneous assessment of performance and condition monitoring is becoming increasingly important. In this respect, digitalization and the accompanying evolution of smart sensors and data acquisition systems give the possibility of applying complex analytics and machine learning algorithms to the compelling need of engine performance monitoring and failure identification. The present paper proposes a solution to the problem of condition monitoring using a method of statistical analysis of multidimensional information acquired from the sensors. The factor analysis method is used to derive a performance index showing engine state deviation from the normal condition. At the same time, principal factor loadings are used as features characterizing the contribution of every measured and analyzed parameter to the variance of the performance index. The benchmark of the developed method is illustrated using the simulation model of a diesel engine with incorporated models of failure states. The latter was developed and validated from the data measured on the test engine.
\end{abstract}

\section{Introduction}

With hardened competition and stringent regulations in the shipping industry, stronger demand for greater efficiency, reduced emissions, and increased profitability becomes evident. In response to this, the ship and propulsion system designs are characterized by continuously rising complexity. As a result, engine manufacturers introduce new engine designs that are compatible with a wide variety of fuels and equipped with intelligent electronic systems providing flexible control and online tuning. Simultaneously, the evolution of intelligent sensors and data acquisition systems and advanced telecommunications fostered the equipment manufacturers and service providers in the shipping industry to constantly implement digitalization and computerization to encapsulate and transfer an analog world to a digitalized world. Digitalization is one of the technological improvements, which is also referred to as digital shipping, is expected to increase ship efficient utilization. On the other hand, digitalization contributes to reducing the number of crew members while bringing a significant increase in

\footnotetext{
* Received March 31, 2021

*** Member, National Maritime Research Institute

**** Mitsui E\&S Machinery
}

the workload of monitoring and troubleshooting tasks [1]. Thus, the condition monitoring and unsupervised processing of the propulsion system operating parameters, creating value for the operators, is of great importance.

While the condition monitoring and information processing algorithms have been widely used in such sectors as aviation and nuclear power plants, there are not many applications in the marine field. Nevertheless, several applications have been made in the past few years. For example, WinGD has proposed a WiDE (WinGD Integral Digital Expert) solution [2] that consists of data acquisition, engine status visualization and diagnosis systems. The ClassNK introduced a set of consulting services called CMAXS for the maintenance and management of onboard equipment. Among them, e-GICSX is aimed at automatic diagnostic of propulsion system abnormal state [3].

In condition monitoring, the development of a suitable model of the target system is a primary task allowing for data classification with respect to the reference (nominal) conditions. In recent times, machine-learning (ML) methods are widely implemented for the purpose of monitoring and 
diagnostic tasks. The ML model learns from the data provided to identify normal or abnormal engine behavior and then characterizes the current state. Thus, in the literature [4], the performance and potentialities of the regularized least square (RLS) and support vector machine (SVM) models were benchmarked by training them on data to forecast the performance decay of propulsion system on a naval vessel. Likewise, in the literature [5], a robust framework for analyzing performance data and condition monitoring was constructed. First, a one-class SVM classifier was trained to represent a nominal state, and then a healthiness index is represented by the probability of each new point belonging to the same performance profile with training data. The data-driven approaches, however, provided limited solutions for specific machinery in specific operational conditions. What is more, a large amount of quality labeled data is required to generalize the model for every case being examined, including data from different operational profiles of the ship and/or failure rates.

In the meantime, however, satisfactory results can also be achieved using the methods of statistical analysis of multidimensional information. In this paper, the application of the method of factor analysis to the problem of propulsion system condition monitoring is scrutinized closely. Following the method, the covariance matrix of parameters deviation from the nominal state is decomposed to a set of factor loadings components. The latter provides the calculation of the relative contribution to the explained variance, which is used as a condition state indicator. The method is benchmarked on data obtained from the propulsion system simulator with the incorporated degradation models. The following chapters detail the method and the failure simulator.

\section{Propulsion System Simulator}

\subsection{Introduction}

A generic ship propulsion system may be considered made up of three main components: the engine, the propeller and the hull. However, in the present study, the application of the condition monitoring method is limited to the engine systems only. What is more, the data required to validate the simulator and degradation models were obtained from the testbed engine. Thus, the propulsion system is referred to as the testbed engine loaded by a water brake.

For the dynamic engine model formulation, a hybrid cycle-mean value (H-CMV) modeling approach is used [6, 7]. The H-CMV approach consists in combining the continuous cycle-averaged model of air and exhaust gases flows through the engine with the discrete consideration of the combustion cycle in the cylinder. Figure 1 illustrates the outline of the engine model. The model is structured in a modular arrangement. Every entity represents an essential component of the engine, such as the compressor, air cooler, air receiver, exhaust gas receiver, turbine and cylinder unit, together with the shaft dynamics. The steady-state performance maps, time-depended momentum, energy and mass equations, and non-linear algebraic equations are used to describe the component's behavior.

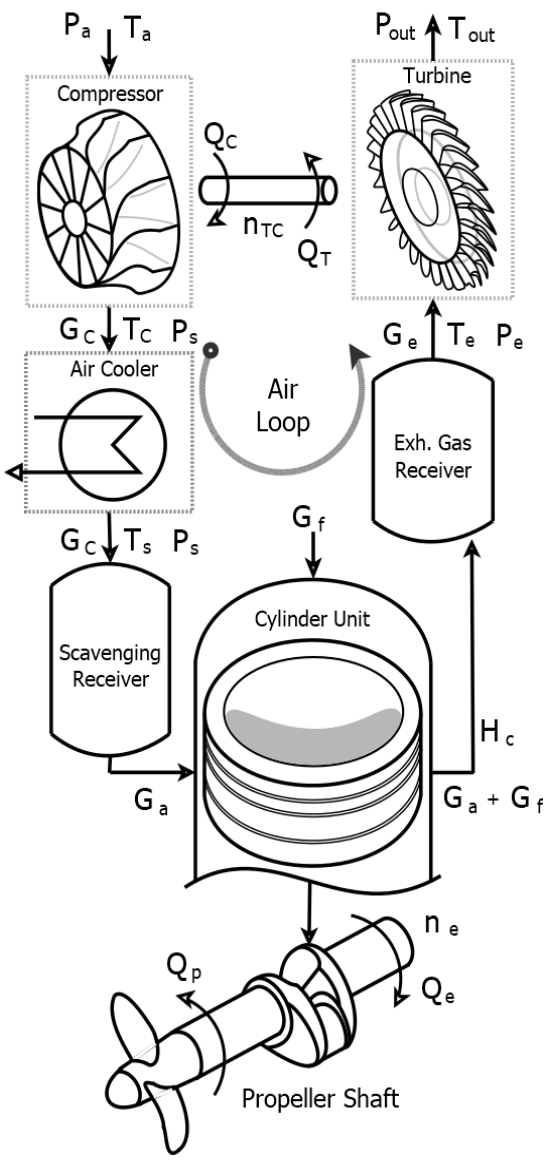

Fig. 1 Composition of a generic marine diesel engine for $\mathrm{H}-\mathrm{CMV}$ modeling

The key differential equations describing both dynamic and thermodynamic engine behavior are based on the laws of conservation for mass and 
energy:

$\frac{d P}{d t}=\frac{R T}{V}\left(G_{\text {in }}-G_{\text {out }}\right)$

$C_{v} M \frac{d T}{d t}+u \frac{d M}{d t}=\left(H_{\text {in }}-H_{\text {out }}\right)$,

where $G_{\text {in }}$ and $G_{\text {out }}$ are the inlet and outlet mass flow rates, respectively; while $H_{\text {in }}$ and $H_{\text {out }}$ are inlet and outlet energy rate, respectively; $V$ is the component volume; $M$ and $u$ are the mass and internal energy, respectively; while $P$ and $T$ are the pressure and temperature, respectively.

The dynamics of the rotating shaft is determined by the momentum conservation law:

$\frac{d n}{d t}=\frac{1}{2 \pi I_{s h}}\left(Q_{e}-Q_{l}\right)$

where $n$ is the shaft rotational speed, $I_{s h}$ is the inertia moment of the whole shaft line; $Q_{e}$ and $Q_{1}$ are the engine torque and load torque, either propeller or water-brake.

Since the attention is focused on the degradation models of particular components, a detailed discussion on the complete engine model is omitted for this paper. However, full description and model validation for a low-speed two-stroke engine can be found in [7].

\subsection{Components Degradation Models}

Two types of faults can be recognized in the propulsion system: the abrupt faults, which can only be discovered at the time of occurrence and consequently are not identifiable by the condition monitoring; the incipient faults are characterized by the time-dependent degradation of components performance. The latter is highly suitable for identification by condition monitoring. In order to develop and study the characteristics of the condition monitoring system, the model of the propulsion system described in the preceding chapter has been updated to take into account the performance degradation of several components.

One cannot deny that the compressor's performance, air cooler (AC) and turbine, which forms the engine air loop, affects the entire propulsion system's performance. Thus, faults related to the air loop are considered in this paper. These are clogging of compressor air filter, fouling of air cooler, and fouling of the exhaust duct after the turbine.

\subsubsection{Air filter clogging}

A turbocharger provides the air supply to the engine cylinder for the combustion of fuel. The dynamics of the turbocharger (TCH) with respect to rotational speed is represented in the following form:

$\frac{d n_{t c}}{d t}=\frac{1}{2 \pi I_{t c}}\left(Q_{T}-Q_{C}\right)$,

where $Q_{T}$ is the torque developed by the turbine due to the expansion of exhaust gas, $Q_{C}$ is the torque required by the compressor to compress the air mass flow $G_{c}$ from the pressure after filter $P_{a f}$ to pressure $P_{S}$ in scavenging receiver, $n_{t c}$ is the turbocharger rotational speed, $I_{t c}$ is the inertia of the turbocharger shaft.

The isentropic work required for an air pressure increase in a compressor, appropriately modified by the efficiency $\eta_{i c}$, yields the following expression for compressor torque:

$Q_{C}=\frac{C_{p_{a}} T_{a} G_{c}}{2 \pi n_{t c} \eta_{i_{C}}}\left(\left\{\frac{P_{s}}{P_{a f}}\right\}^{\frac{k_{a}-1}{k_{a}}}-1\right)$,

where $C_{p a}$ is the specific heat constant of air, $k_{a}$ is the ratio of the specific heat for air, $P_{a f}$ is the pressure after the filter.

The pressure drop, $\Delta P_{a f}$, through the air filter is proportional to the square of the air mass flow in the following form:

$\Delta P_{a f}=\frac{\xi_{a f} G_{c}^{2}}{\rho_{a}}$,

where $\xi_{a f}$ is the flow coefficient, $\rho_{a}$ is the air density.

The air filter clogging is introduced as a degradation function [8] of the flow coefficient in the following form:

$\xi_{a f}(t)=k\left\{1-e^{\frac{t}{\tau_{d}}}\right\}$,

where $k, \tau_{d}$ are the decay coefficient and time constant, respectively.

2.2.2 Air cooler fouling

In order to keep the density of air after the compressor as high as possible, an air cooler is installed before the engine cylinder's input. The temperature of the air after AC is modeled as follows:

$T_{s}=T_{c w}+\eta_{a c}\left(T_{c}-T_{c w}\right)\left(\frac{G_{c}}{G_{c_{m c r}}}\right)^{\frac{1}{3}}$, 
where $T_{c w}$ is the water temperature at the cooler inlet, $\eta_{a c}$ is the cooler effectiveness, $T_{c}$ is the air temperature after the compressor.

The fouling of $\mathrm{AC}$ can be caused by air impurities that, together with oil vapors, produce a layer covering the surface on the airside. From the waterside, the scale can form a layer of insulation. The main consequence is the degradation of $\mathrm{AC}$ effectiveness and the rise of outlet temperature. Thus, the AC fouling is introduced as a degradation function of the cooler effectiveness in the following form:

$$
\eta_{a c}(t)=k\left\{1-e^{\frac{t}{\tau_{d}}}\right\} \text {. }
$$

\subsubsection{Exhaust duct fouling}

From the propulsion system efficiency point of view, the exhaust gas energy recovery after the turbine is commonly applied by installing a steam boiler. Also, more and more ships are being equipped with selective catalytic reduction (SCR) systems. Therefore, fouling can be caused by soot in the exhaust gases and oil vapors that produce layers covering the surfaces of boilers and SCR. The main consequence is the degradation of TCH performance due to increased backpressure after the turbine.

The isentropic work of gas expansion in the turbine appropriately modified by the efficiency $\eta_{i t}$ yield the expression for turbine torque:

$$
Q_{T}=\frac{C_{p_{e}} T_{e} G_{e} \eta_{i_{T}}}{2 \pi n_{t c}}\left(1-\left\{\frac{P_{o}}{P_{e}}\right\}^{\frac{k_{e}-1}{k_{e}}}\right),
$$

where $C_{p e}$ is the specific heat constant of gas, $k_{e}$ is the ratio of the specific heat for gas, $G_{e}$ is the exhaust mass flow, $P_{o}$ is the pressure after the turbine.

Likewise, the air filter clogging, the exhaust duct fouling is introduced as a degradation function of the flow coefficient in the following form:

$$
\Delta P_{o}=\frac{\xi_{e d} G_{e}^{2}}{\rho_{e}}, \quad \xi_{e d}(t)=k\left\{1-e^{\frac{t}{\tau_{d}}}\right\},
$$

where $\Delta P_{o}$ is the pressure drop through the duct, $\xi_{e d}$ is the flow coefficient, $\rho_{e}$ is the gas density.

The parameters of the presented above degradation functions can be empirically derived from the available historical data. However, such data is not usually available, and thus the particular experiment on the test engine was set up to identify the degradation model parameters.

\subsection{Identification and Validation of Degradation Models}

The actual degradation behavior of the engine components is both time and operation profile-dependent, and thus a vast combination of conditions is required. However, for the purpose of the present research, not the degradation behavior but the validity of the simulation model response to the degradation of the components is of high relevance.

The experiments were performed on the two-stroke marine diesel test engine kindly provided by Mitsui E\&S Machinery. Table 1 summarizes the specification of the test engine. The engine was run at a constant speed and load corresponding to $75 \%$ of the Maximum Continuous Rating (MCR). The previously described degradation models were introduced as follows: exhaust duct fouling was imitated by gradually closing a valve on the exhaust stack of the test engine; the AC fouling was imitated by gradually reducing the flow of cooling water through the cooler. The air filter clogging imitation was considered the most complicated due to the risk of the compressor surging. Thus, the degradation was introduced in the reverse order. Engine load was reduced to $25 \%$ MCR, the air filter was partially closed with a cover, engine load was returned to $75 \% \mathrm{MCR}$, and then the cover was gradually sliding.

The degradation functions, introduced by Eqs. (6), (8) and (10), are essentially continuous in time; nonetheless, the degradations were introduced in a stepwise discrete fashion, as illustrated in Fig.2.

The first step towards the validation of the engine simulation model with introduced failures is the identification of degradation models introduced on the test engine. Figure 3 shows the time evolution of parameter degradation measured on the test engine and the model identification results. After successfully identifying the degradation, the second step compares the engine simulation model responses with those measured on the test engine under similar conditions. The results of engine 
model responses are depicted in Fig. 4. As can be seen, although there are some biases between measured and simulated state variables, the engine model correctly reproduces the responses of the test engine to all the failure models introduced. Therefore, the engine simulation model can be used as an equivalent substitute for the actual engine with failures.

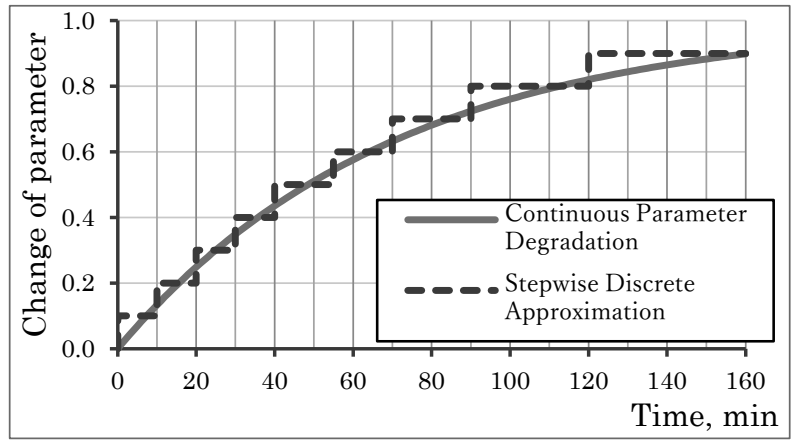

Fig. 2 Stepwise approximation of a continuous function

Table 1. Mitsui-MAN engine specification at MCR

\begin{tabular}{|l|l|}
\hline Engine Type & 4S50ME-T9 \\
\hline No of Cylinders & 4 \\
\hline Bore/Stroke, $[\mathrm{mm} / \mathrm{mm}]$ & $500 / 2214$ \\
\hline Power, $[\mathrm{kW}]$ & 7120 \\
\hline Speed, [rpm] & 117 \\
\hline IMEP, [bar] & 22 \\
\hline
\end{tabular}
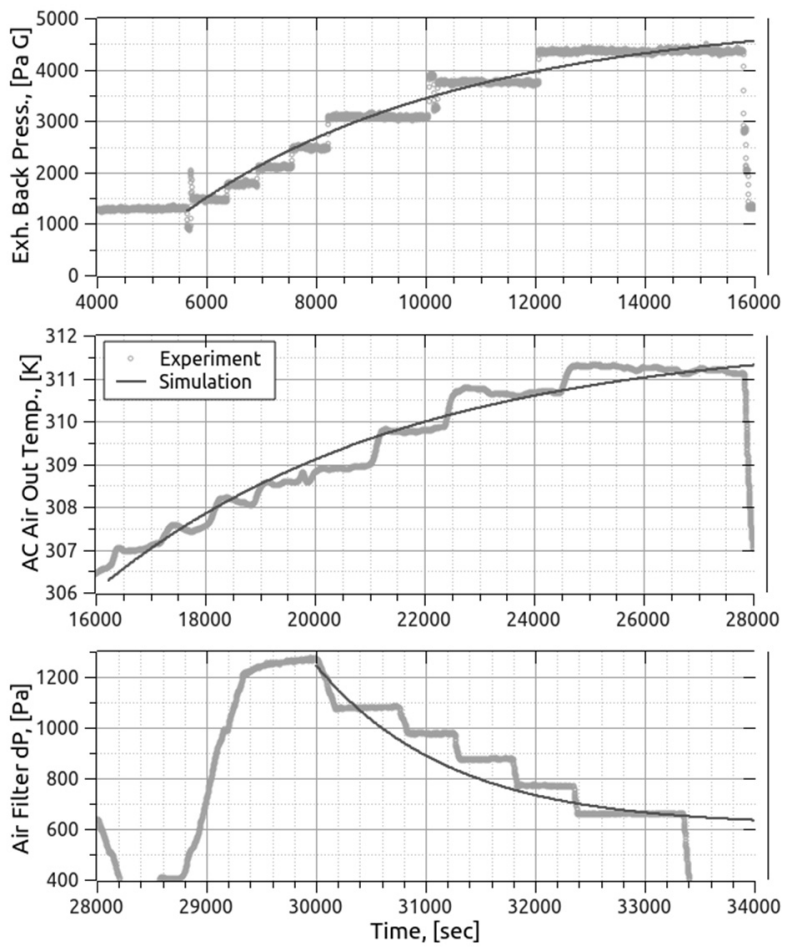

Fig. 3 Degradation models identification results
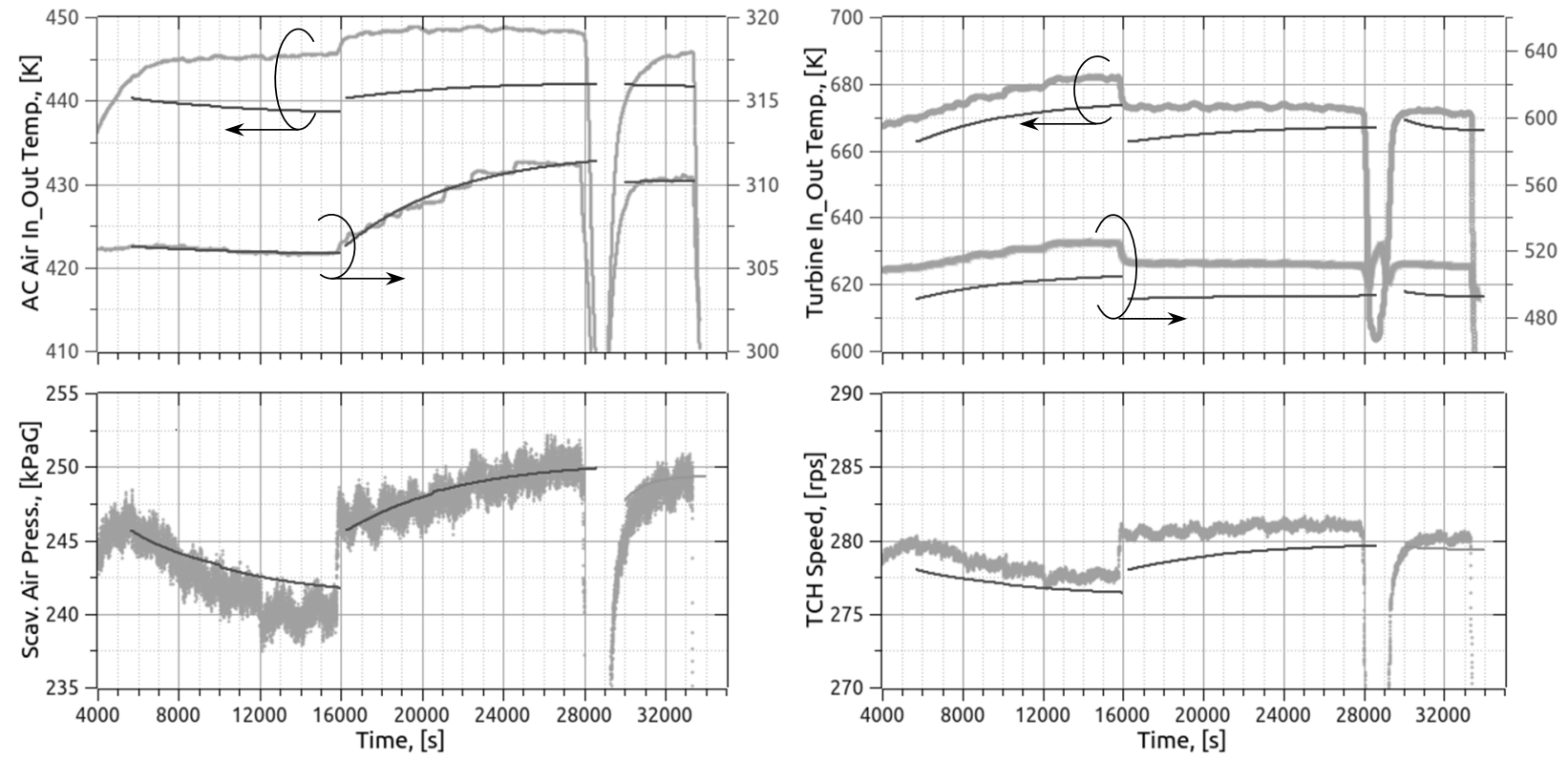

Fig. 4 Validation of the engine simulation model responses to the introduced failure events 


\section{Condition Monitoring Framework}

\subsection{Method of Factor Analysis}

The statistical method of factor analysis (FA) plays a central role in extracting non-trivial information from a set of raw data collected from sensors of the monitored component. FA is the method designed to analyze interrelationships within a set of variables. The critical assumption of the method is that the measured variables can be correlated in such a way that their correlation may be reconstructed by a few (latent) common parameters, called factors. These factors, in turn, could represent the underlying structure and inherent interdependencies in a concise and interpretable form [9, 10].

The general factor model can be written as $\boldsymbol{Y}=\boldsymbol{A} \boldsymbol{F}+\boldsymbol{\Psi}$,

where $\boldsymbol{Y}=\left(y 1, y_{2}, \ldots, y_{p}\right)^{\mathrm{T}}$ is a vector of measured variables, $\boldsymbol{F}=\left(f_{1}, f_{2}, \ldots, f_{i}\right)^{\mathrm{T}}$ is a vector of $r<p$ latent variables or factors, $A$ is a ( $p \times r)$ matrix of fixed coefficients (factor loadings) and $\boldsymbol{\Psi}=\operatorname{diag}\left(u_{1}\right.$, $\left.u_{2}, \ldots, u_{p}\right)$ is a diagonal matrix of random error terms. The latter consists of errors of measurements, together with unique individual effects associated with each variable $y_{i}$. Figure 5 demonstrates the relationships among variables in the factor model.

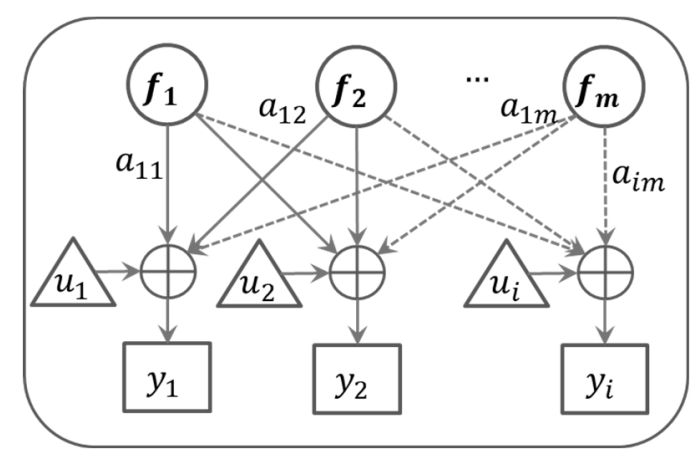

Fig. 5 Representation of factor relationships

\subsection{Algorithm of Factor Analysis}

The FA algorithm analyses the correlations among the parameter deviations from the base (nominal) level of monitored parameters. The latter can be provided from the accompanying nominal state simulation model (digital twin), or some initial set of parameters can be considered as a base level. For the sake of brevity, only brief details of the algorithm are given below, and full theoretical background can be found in $[9,10]$.

Factor analysis can be viewed as a particular case of the weighted principal components model, and thus principal components are used to estimate the factors.

The correlation matrix for a set of $p$ measured parameters during a time of length $N$ is given as:

$\boldsymbol{R}=\frac{\boldsymbol{Y}^{T} \boldsymbol{Y}}{N}$

$Y_{i, j}=\frac{X_{i, j}-\bar{X}^{\langle j\rangle}}{\sigma_{X^{\langle j\rangle}}}, \quad i=1, \ldots, N, j=1, \ldots, p$

where $\boldsymbol{Y}$ is a matrix $(N \times p)$ of scaled parameter deviations from the base level, $\sigma_{x}$ is the parameter standard deviation.

In turn, the ( $p \times p)$ correlation matrix $\boldsymbol{R}$ can be expressed as the sum of $p$-eigenvalues multiplied by their eigenvectors and their transpose that is a singular value decomposition (SVD) of matrix $\boldsymbol{R}$.

$\boldsymbol{R}=\boldsymbol{U} \boldsymbol{S} \boldsymbol{V}^{T}$,

Eventually, the estimator of the factor loadings matrix is defined as:

$\boldsymbol{A}=\boldsymbol{U} \sqrt{\boldsymbol{S}}$,

The principal components analysis gives a set of axes to which variables could be referred, and thus the factor loadings are the projections of the variables onto the factor axes. Although mathematically unique, a set of factors is the only one of an infinite number of sets that describes data configuration just as well. Still, it does not guarantee maximum statistical significance. Therefore, as the second step of the analysis, the maximum likelihood principle can be used to specify the elements of matrix $\boldsymbol{A}$ that best model the correlation structure of data $\boldsymbol{Y}$.

In the beginning, the factors $\boldsymbol{F}$ and data $\boldsymbol{Y}$ are assumed as a joint Gaussian distribution:

$$
\left[\begin{array}{l}
\boldsymbol{Y} \\
\boldsymbol{F}
\end{array}\right] \backsim\left(\left[\begin{array}{l}
0 \\
0
\end{array}\right],\left[\begin{array}{cc}
\boldsymbol{A} \boldsymbol{A}^{T}+\boldsymbol{\Psi} & \boldsymbol{A} \\
\boldsymbol{A}^{T} & \boldsymbol{I}
\end{array}\right]\right),
$$

To maximize the parameters of the joint distribution above, the log-likelihood function of the parameters yields:

$$
\ell(\boldsymbol{A}, \boldsymbol{\Psi})=\sum E\left[\begin{array}{l}
-\frac{1}{2} \log \boldsymbol{\Psi}-\frac{N}{2} \log 2 \pi \ldots \\
-\frac{1}{2}(\boldsymbol{Y}-\boldsymbol{A} \boldsymbol{F})^{T} \boldsymbol{\Psi}^{-1}(\boldsymbol{Y}-\boldsymbol{A F})
\end{array}\right],
$$


The maximization of the likelihood function with respect to the matrix $A$, is performed by applying the iterative Expectation-Maximization (EM) algorithm. For the sake of brevity, the details of the algorithm are omitted and can be found in [11].

Although, as was mentioned earlier, the factors contain the essential information related to the variance of measured parameters, the factors loadings are considered more informative for the condition monitoring purpose. This is because the components of factor loadings matrix $A$ reflect the degree of contribution of every parameter deviation onto the explained variance of every factor. Thus, a performance index of the component condition is defined as the relative share of the explained variance formed by the factors loadings of the measured parameters:

$$
D^{\langle n\rangle} \equiv \sigma^{2}\left[F^{\langle n\rangle}\right]=\frac{\sum_{i=1}^{p} a_{i, n}^{2}}{\sum_{n=1}^{N} \sum_{i=1}^{p} a_{i, n}^{2}}, \quad n=1, \ldots, N
$$

As one may note, the number of performance indices is equal to the number of processed parameters $p$, and this fact does not facilitate efficient condition monitoring. Simultaneously, the eigenvalues of the correlation matrix $R$ usually decay rapidly, which suggests that only a few first components of $\boldsymbol{R}$ and thus a few first factors can be considered as principal containing a significant share of the explained variance. Therefore, $N$ in Eq. (17) is limited to $N \leq 2$.

As mentioned above, the factor loadings indicate the relationship strength between parameters and factors variation. Therefore, this property can be considered a feature that characterizes every measured parameter's contribution to the variance of the performance index. Thus, the relative contribution of loadings to the variance of factors is given as:

$$
\lambda_{i, n}=\frac{a_{i, n}^{2}}{\sum_{i=1}^{p} a_{i, n}^{2}}, \quad n=1,2
$$

\subsection{Application of FA to Condition Monitoring}

This section presents a case study demonstrating the FA methodology implementation to the condition monitoring framework. The analysis applied to the data obtained from the developed simulator of the engine with fault models. In order to demonstrate the qualitative difference of the results and due to limitations of paper length, only the cases of air filter clogging and $\mathrm{AC}$ fouling are demonstrated and discussed.

The engine simulation model was set to run at a load corresponding to $75 \%$ MCR and speed 106 rpm. The steady-state simulation is followed by the response of engine state variables to the introduced incipient failure of air filter clogging. The time constant in the degradation model is set to $\tau_{d}=3600$ sec. The fault is introduced at $\mathrm{T}=1800 \mathrm{sec}$. Figure 6 illustrates the engine state variables time evolution. The sensors noise imitation is also added to the results. The parameters listed in Table 2 were recorded every 1 sec.

Table 2. List of recorded parameters

\begin{tabular}{|l|l|}
\hline Engine Speed, [rpm] & $\mathrm{Ne}$ \\
\hline TCH Speed, [rps] & $\mathrm{Ntc}$ \\
\hline Scav. Air Pressure, [kPa] & $\mathrm{Ps}$ \\
\hline Scav. Air Temperature, [K] & $\mathrm{Ts}$ \\
\hline Exh. Gas Pressure, [kPa] & $\mathrm{Pe}$ \\
\hline Exh. Gas Temperature, [K] & $\mathrm{Te}$ \\
\hline Maximum Compression Pressure, [bar] & $\mathrm{Pc}$ \\
\hline Maximum Combustion Pressure, [bar] & $\mathrm{Pz}$ \\
\hline
\end{tabular}

The FA algorithm is applied in a discrete fashion as follows:

- Select the array of recorded parameters $(N=$ 600-time steps)

- Calculate correlation matrix $\boldsymbol{R}$ (Eq. (12))

- Estimate matrix of factor loadings $A$ (Eq. (14))

- Refining elements of $\boldsymbol{A}$ with EM algorithm (Eq. (16))

- Evaluate performance index $D_{1}$ for the principal factor

- Evaluate the relative contribution of factor loadings $\lambda_{\mathrm{i}, 1}$ (Eq. (18))

- Slide the analysis window to get a new set of recorded parameters $(N=N+\Delta t, \Delta t=150)$

Figure 7 illustrates the relative contribution of parameter variation to the variation of the performance index. Regarding the considered failures, as one may note, there is a qualitative difference in the factor loadings patterns, implying that every failure may have a unique set of features. 
At the same time, Figure 8 shows the evolution of performance indices $D_{1}$. As can be seen, both indices clearly indicate the onset of an abnormal engine
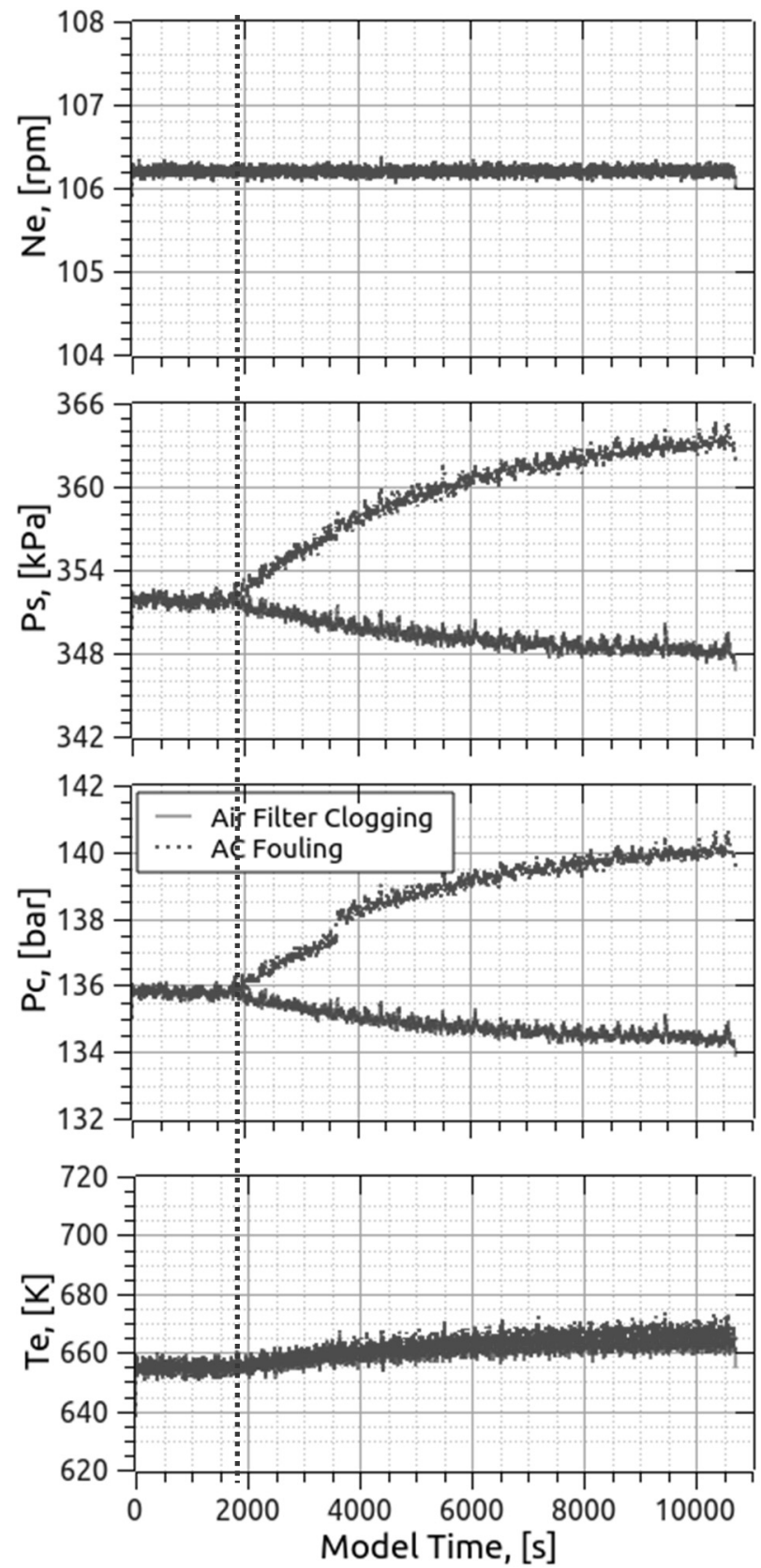

state which corresponds to a very early moment of degradation development.
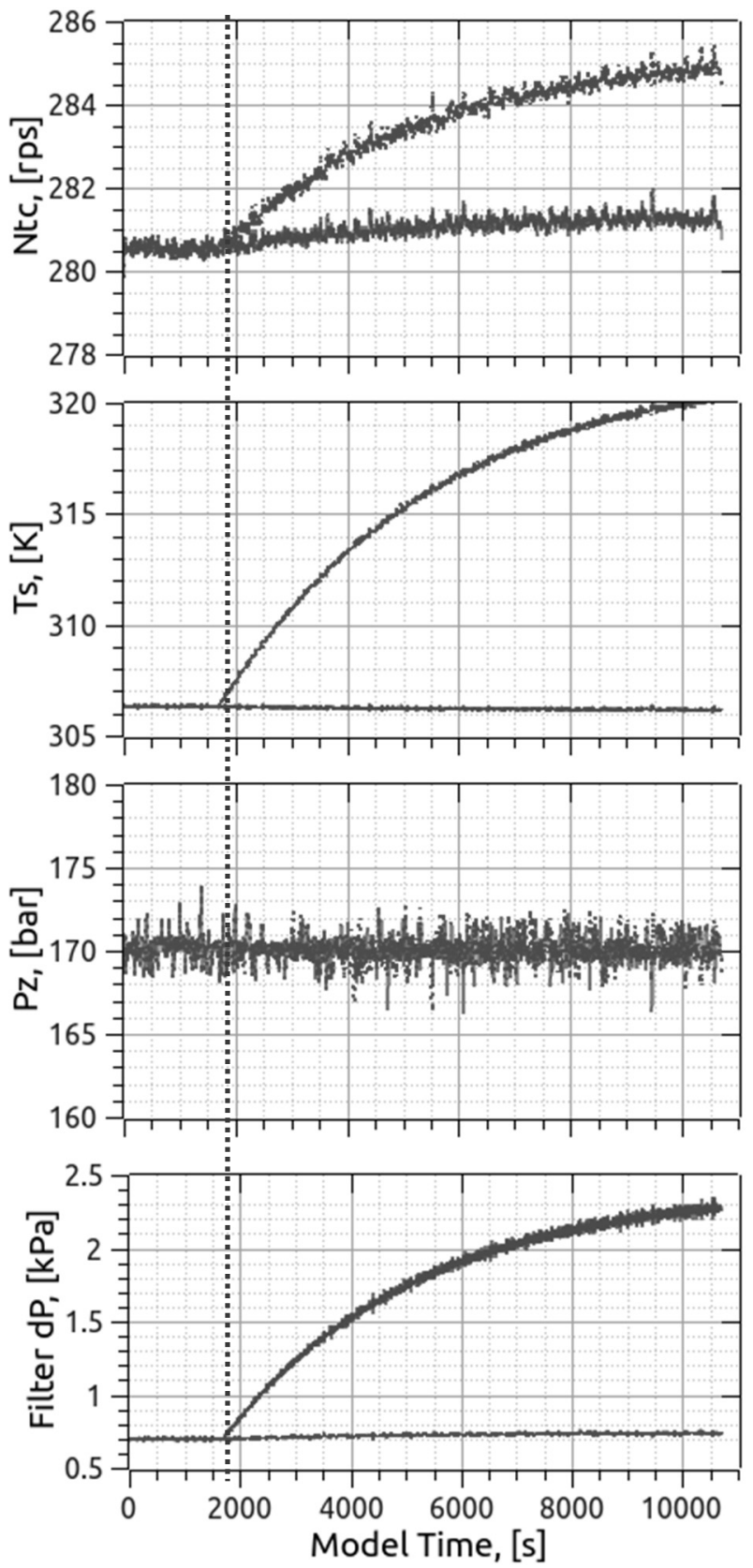

Fig.6 Engine simulation model responses to the air filter clogging and AC fouling faults 

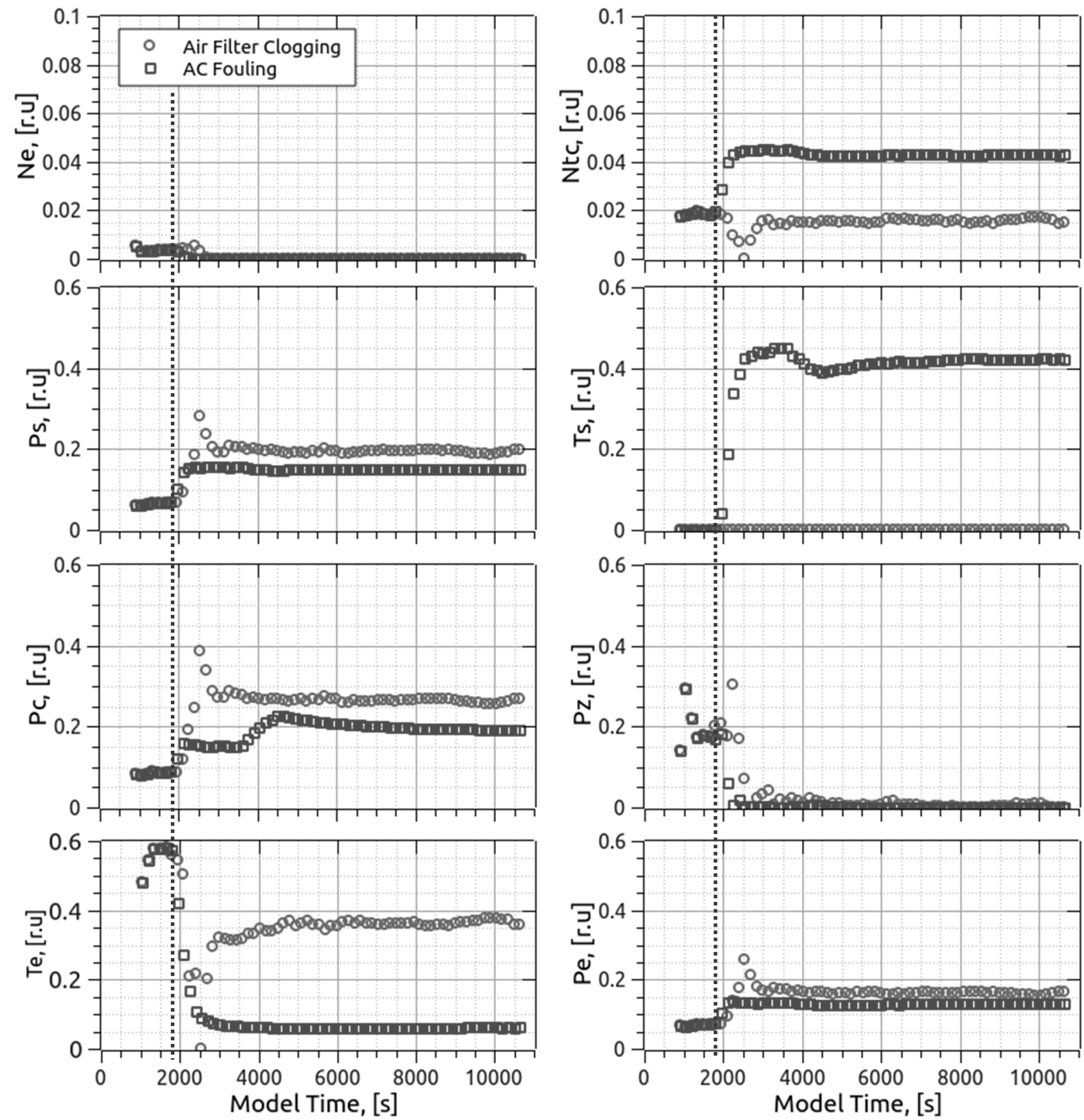

Fig.7 Relative contribution of measured parameters factor loadings to the variance of principal factor $F_{1}$ 


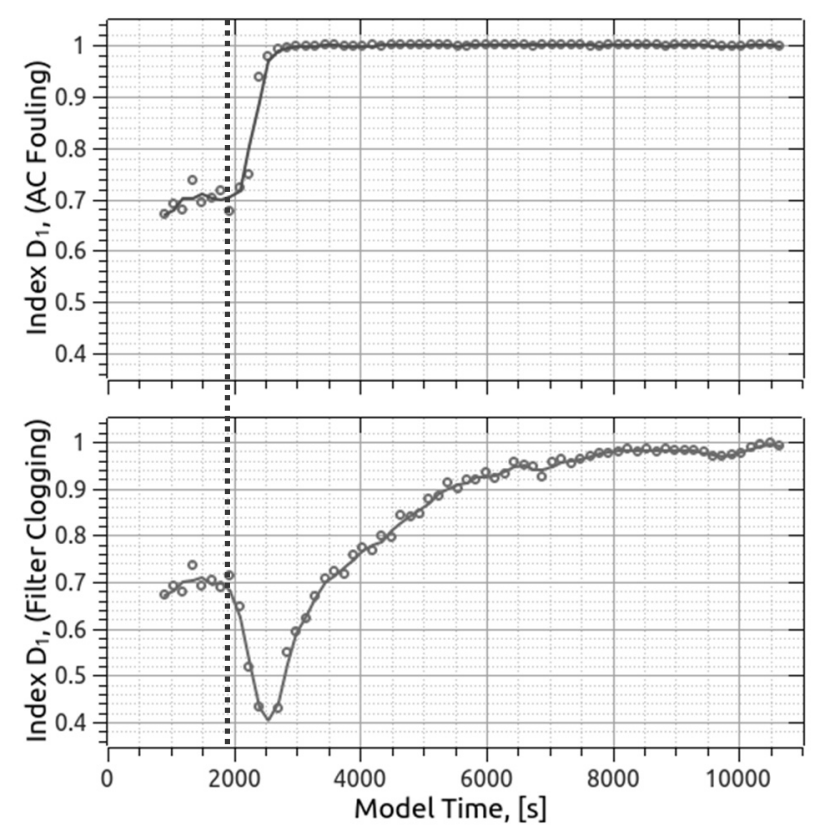

Fig.8 The performance indices $D_{1}$ of the engine condition

\section{Conclusion}

The proposed framework for propulsion system condition monitoring is based on the statistical method of factor analysis and proved its effectiveness. The performance index provides for efficient recognition of failure event which occurs in the monitored component. Moreover, the performance index clearly shows the onset of the failure event, providing early detection of degradation even in case of a minor change of the recorded parameters.

Furthermore, the incipient failures cause deviation of a group of the engine parameters, such as TCH speed, scavenging air pressure, exhaust gas temperature, etc. However, these deviations are usually of minor significance far from the alarm monitor system thresholds. In this respect, the relative contributions of parameter factor loadings to the principal factor variance clearly show the anomaly pattern and are unique for every failure. Thus the latter can be used as a set of features characterizing the failure, which are highly suitable for the ML algorithms such as clustering and classification. In turn, the ML algorithms can identify the cause of engine performance degradation, and such algorithms are currently under development.

\section{Reference}

1) Man, Y., Lundh, M. \& MacKinnon, S.N. Managing unruly technologies in the engine control room: from problem patching to an architectural thinking and standardization. WMU J Marit. Affairs Vol.:17, pp.497-519 (2018).

2) WinGD: WiDE WinGD Integrated Digital Expert. https://www.wingd.com/getattachment/Digital-solutio ns/WiDE/WiDE_Brochure_8pp_FINAL.pdf/?lang=enGB

3) Introduction of ship maintenance management system ClassNK CMAXS (in Japanese), https://classnkcs.co.jp/archive/pdf/seminar01.pdf

4) Coraddu A, Oneto L, Ghio A, Savio S, Anguita D, Figari M. Machine learning approaches for improving condition-based maintenance of naval propulsion plants. In Proc: the Institution of Mechanical Engineers, Part M: J. of Eng. for the Mar. Env. 2016; 230(1): 136-153.

5) Gkerekos C., Lazakis I., and Theotokatos G. Ship Machinery Condition Monitoring Using Performance Data Through Supervised Learning. In Proc.: Smart Ship Technology Conference. Royal Institute of Naval Architects, London, (2017), pp.105-111

6) Baldi F, Theotokatos G, Andersson K. Development of a combined mean value-zero dimensional model and application for a large marine four-stroke diesel engine simulation. Appl Energy 2015;154:402-15.

7) Bondarenko O., Fukuda T. (2020) Development of a diesel engine's digital twin for predicting propulsion system dynamics. Energy, Vol.196.

8) Ken D.Q., Seaton R.E. A theoretical analysis of thermal surface fouling. Br Chem. Eng. (1959); 4(5), pp.258-262.

9) Harman H.H. Modern Factor Analysis. Second Edition. The University Chicago Press. (1968)

10) Cureton E.E., D'Agostino R.B. Factor Analysis, An Applied Approach. Psychology Press. (1983)

11) Basilevsky A. Statistical Factor Analysis and Related Methods, Theory and Application. A Wiley-Interscience Publication. (1993) 